\title{
Impact of High-Sensitivity Cardiac Troponin Elevation in Relation to Diagnostic Invasive Intravascular Imaging for the Assessment of Coronary Artery Disease
}

\author{
Yoshinori Sanuki, ${ }^{1}$ MD, Shinjo Sonoda, ${ }^{1}$ MD, Yoshitaka Muraoka, ${ }^{1}$ MD, Konosuke Inoue, ${ }^{1}$ MD, \\ Koshi Setoyama, ${ }^{1}$ MD, Toshiya Miura, ${ }^{1}$ MD, Akiyoshi Shimizu, ${ }^{1}$ MD, Reo Anai, ${ }^{1}$ MD, \\ Tetsu Miyamoto, ${ }^{1}$ MD, Yasushi Oginosawa, ${ }^{1}$ MD, Yuki Tsuda, ${ }^{1}$ MD, \\ Masaru Araki, ${ }^{1}$ MD and Yutaka Otsuji, ${ }^{1} \mathrm{MD}$
}

\begin{abstract}
Summary
Recent studies reported that cardiac troponin elevation after percutaneous coronary intervention is related to adverse cardiac events. Intravascular ultrasound (IVUS) and optical coherence tomography (OCT) are often used to assess lesion characteristics in the coronary arteries. However, little is known about the trend of cardiac troponin elevation after diagnostic invasive intracoronary examination and the prognostic influence. We assessed the relationship between myocardial injury manifested by the high-sensitivity cardiac troponin $\mathrm{T}$ (hs-cTnT) level after invasive intracoronary examination and future adverse cardiac outcomes. We evaluated 115 patients with stable coronary artery disease who underwent IVUS or OCT for detailed coronary assessment during coronary angiography (CAG). Baseline and post-procedural (within 24 hours after examination) hs-cTnT were measured. In consequence, post-procedural hs-cTnT level and percentage increase were higher in patients with IVUS or OCT during CAG than in those without. Periprocedural myocardial injury (PMI, defined as post-procedural hscTnT with upper reference limit greater than five-fold) occurred in $10(8.6 \%)$ patients. There were no significant differences in baseline characteristics between patients with and without PMI, except for left-ventricular diastolic dimension. Only two major adverse cardiac events (MACE, defined as cardiovascular death, nonfatal myocardial infarction, and target lesion revascularization) occurred in non-PMI during a mean observation period of $32 \pm 18$ months. On Kaplan-Meier analysis, MACE-free survival rate was similar between PMI and non-PMI. In conclusion, a few imperceptible PMI derived by hs-cTnT assay occurred after diagnostic invasive intracoronary examination. However, it was not associated with subsequent poor cardiac outcome.
\end{abstract}

(Int Heart J 2019; 60: 601-607)

Key words: Intravascular ultrasound, Optical coherence tomography, Adverse cardiac events, Periprocedural myocardial injury, Diagnostic coronary angiography

I ntravascular ultrasound (IVUS) and optical coherence tomography (OCT) have recently been used more often to assess lesion severity and plaque morphology in patients with coronary artery disease. Several studies have revealed the short- and long-term safety of IVUS and OCT. ${ }^{1,2)}$ Cardiac troponin is a biochemical marker of myocardial necrosis. Omland et al. have shown that highsensitivity cardiac troponin concentrations in patients with stable coronary artery disease are associated with adverse cardiac outcomes. ${ }^{3)}$ Other studies have demonstrated that periprocedural myocardial injury (PMI), defined as a cardiac troponin elevation after percutaneous coronary intervention (PCI), was also related to adverse cardiac events. ${ }^{4 \cdot 6)}$ We have recently reported elevation of the highsensitivity cardiac troponin $\mathrm{T}$ (hs-cTnT) level in patients who underwent diagnostic coronary angiography (CAG) with additional coronary assessment using IVUS.? This finding suggests that IVUS might cause myocardial injury. However, little is known about the clinical significance of hs-cTnT elevation after IVUS assessment during diagnostic CAG. Furthermore, no reports have described the influence of OCT on the results of cardiac troponin assays. The aim of this study is to assess the incidence of myocardial injury manifested by the hs-cTnT level after invasive intracoronary examination (IVUS and OCT) and determine the relationship between such myocardial injury and subsequent adverse cardiac outcomes.

\section{Methods}

Study population: This retrospective study was conducted in our institution from August 2010 to December 2015. All patients underwent invasive intracoronary examination with IVUS or OCT during the study period in

From the ${ }^{1}$ Second Department of Internal Medicine, University of Occupational and Environmental Health, Kitakyusyu, Japan.

Address for correspondence: Shinjo Sonoda, MD, The Second Department of Internal Medicine, University of Occupational and Environmental Health, 1-1 Iseigaoka, Yahatanishi-ku, Kitakyusyu 807-8555, Japan. E-mail: s-sonoda@med.uoeh-u.ac.jp

Received for publication July 24, 2018. Revised and accepted November 19, 2018.

Released in advance online on J-STAGE May 17, 2019.

doi: 10.1536/ihj.18-448

All rights reserved by the International Heart Journal Association. 
order to assess in-stent characteristics of the previously implanted stents or de novo lesions. The exclusion criteria were the operation of PCI or coronary artery bypass surgery immediately after examination, renal insufficiency (serum creatinine of $>2.0 \mathrm{mg} / \mathrm{dL}$ and the need for hemodialysis), the presence of concurrent congestive heart failure before examination, and the presence of acute coronary syndrome within 1 month. The institutional review board of the University of Occupational and Environmental Health approved this study, and all patients provided written informed consent.

CAG: Using a standard technique, a 6-Fr sheath was placed in the radial, femoral, or brachial artery. After administration of intravenous heparin (3,000 IU) and intracardiac nitroglycerin $(0.1-0.2 \mathrm{mg})$, selective left and right $\mathrm{CAG}$ was performed using manual or automatic injection of contrast media. After the IVUS or OCT assessment, final CAG was performed to confirm the lack of angiographically visible acute complications. Target vessel tortuosity, defined as three or more bends with a $\geq 45^{\circ}$ change in vessel direction, was identified. According to the criteria of a previous study, the severity of coronary calcification was also assessed on CAG. ${ }^{8)}$

IVUS and OCT procedure: The major purpose of diagnostic IVUS or OCT examination was for the assessment of in-stent characteristics or de novo significant coronary lesion, which was at the physician's discretion. IVUS or OCT was performed after additional administration of intravenous heparin (2,000 IU) and intracardiac nitroglycerin. A $40-\mathrm{MHz}$ rotational IVUS imaging catheter (Terumo, Tokyo, Japan; Boston Scientific, Natick, MA, USA) was introduced over a 0.014-inch guide wire and automatically retracted at $0.5 \mathrm{~mm} / \mathrm{second}$ from far distal to the aorto-ostial junction of the target lesion. OCT was performed with a time-domain OCT system (M3 system; LightLab Imaging, Westford, MA, USA), a frequencydomain OCT system (C7/C8 system; St. Jude Medical, St. Paul, MN, USA), or an optical frequency-domain system (Lunawave system; Terumo). With the M3 system, an occlusion balloon was advanced proximal to the stenotic lesion over a conventional guide wire. The guide wire was then replaced with an imaging wire, and the balloon was inflated during imaging acquisition. The imaging wire was automatically pulled back at $2.0 \mathrm{~mm} /$ second. During pullback, lactated Ringer's solution was continuously infused from the tip of the occlusion balloon. With the C7/C8 system or Lunawave system, the OCT imaging catheter was advanced distal to the stenotic lesion over the conventional guide wire and automatically pulled back at 20 to $40 \mathrm{~mm} /$ second. During pull-back, contrast medium, or low-molecular-weight dextran was continuously infused from the tip of the guiding catheter.

Data analysis: In our institution, we routinely perform hs-cTnT measurement in the hospital for the assessment of patient risk stratification and occurrence of procedural complication. The hs-cTnT level was checked before and 12 to 24 hours after the examination. PMI was defined as a post-examination hs-cTnT level of $>0.070 \mathrm{ng} / \mathrm{mL}$ (fivefold higher than the 99th percentile of the upper reference limit, $0.014 \mathrm{ng} / \mathrm{mL}){ }^{9)}$ Percentage increase of hs-cTnT (\%) was calculated using the following formula: $100 \times$ (post
hs-cTnT - baseline hs-cTnT)/baseline hs-cTnT. Other laboratory and echocardiographic data were measured before the examination according to previously established procedures.

Follow-up: Major adverse cardiac events (MACE) after the examinations, defined as cardiovascular death, nonfatal myocardial infarction, and target lesion revascularization, were assessed in all patients during a mean observation period of $32 \pm 18$ months (range, 3-67 months). The postdischarge follow-up data were collected at the outpatient clinic or through a telephone interview.

Statistical analysis: Almost all continuous variables were presented as the mean \pm standard deviation. Baseline and post hs-cTnT and percentage increase of hs-cTnT were presented as median values and $95 \%$ confidence interval (CI). All categorical variables were presented as counts and percentages. The overall F-test was reported for the analysis of variance models. Differences between any two groups were assessed with the Chi-squared test for comparisons of categorical variables and nonparametric Wilcoxon test for continuous variables. A $P$-value of $<0.05$ was considered to indicate statistical significance. Statistical analysis was performed using JMP version 9.0 (SAS Institute Inc., Cary, NC, USA).

\section{Results}

Figure 1 presents study flow diagram. In total, 580 patients who underwent IVUS or OCT during diagnostic CAG for the evaluation of in-stent characteristics or de novo significant coronary lesions were assessed in this study. In 424 patients, serial data of hs-cTnT were lacking, and invasive intracoronary assessments with coronary revascularization were performed. After elimination of patients who met the exclusion criteria, 115 patients with stable coronary artery disease were analyzed in this study.

In the study population, diagnostic intracoronary examinations were performed for the assessment of in-stent characteristics at follow-up CAG $(n=86)$, de novo significant coronary lesions $(n=17)$, and both $(n=12)$. In all cases, no complication, such as coronary dissection, coronary spasm, and slow-flow phenomenon, occurred during invasive intracoronary examinations.

Comparison between study population and control group: During the study period, we performed diagnostic CAG with pre- and post-procedural hs-cTnT assessment in 116 patients, after excluding renal insufficiency, the presence of concurrent congestive heart failure, and the presence of acute coronary syndrome within 1 month (control group). No complication occurred in this group during examination. We compared the study population and control group in order to assess the influence of IVUS or OCT on hs-cTnT change after the procedure (Table I). There were no significant differences in baseline characteristics between groups except for history of myocardial infarction and LDL-cholesterol level. Although there were no significant differences in baseline hs-cTnT between groups, post hs-cTnT level and percentage increase of hs-cTnT were significantly larger in the study population than in the control group $[0.019$ (95\% CI 0.016-0.021) versus 0.013 (95\% CI $0.012-0.015) ; 67.0$ 


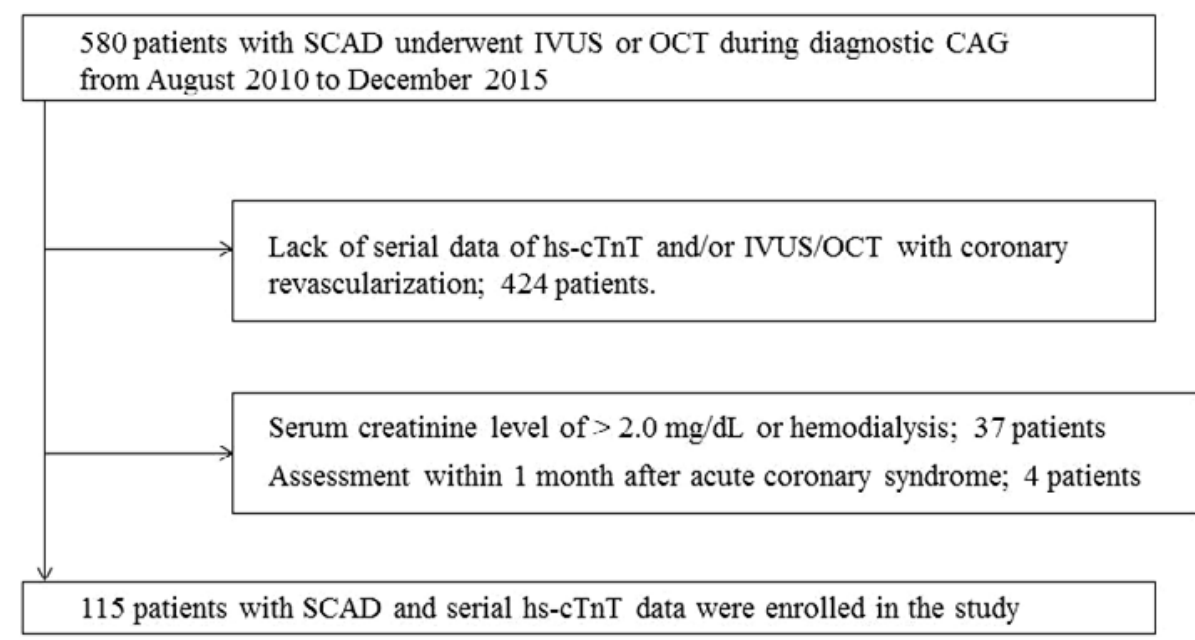

Figure 1. Study flow diagram.

Table I. Comparison Between the Study Population and Control Group

\begin{tabular}{|c|c|c|c|}
\hline & $\begin{array}{l}\text { Study population } \\
\quad(n=115)\end{array}$ & $\begin{array}{l}\text { Control group } \\
\quad(n=116)\end{array}$ & $P$-value \\
\hline Age & $71.5 \pm 9.6$ & $70.8 \pm 9.8$ & 0.62 \\
\hline Male & $77(67)$ & $73(63)$ & 0.52 \\
\hline BMI & $23.3 \pm 3.7$ & $24.6 \pm 4.3$ & 0.64 \\
\hline Diabetes mellitus & $52(45)$ & $60(52)$ & 0.32 \\
\hline Hypertension & $89(77)$ & $92(79)$ & 0.72 \\
\hline Dyslipidemia & $83(72)$ & $73(63)$ & 0.13 \\
\hline Smoke & $68(59)$ & $62(53)$ & 0.38 \\
\hline CKD & $48(42)$ & $44(38)$ & 0.55 \\
\hline OMI & $42(37)$ & $20(17)$ & $<0.01$ \\
\hline $\mathrm{HF}$ requiring treatment & $11(10)$ & $11(9)$ & 0.98 \\
\hline \multicolumn{4}{|l|}{ Laboratory Data } \\
\hline Baseline hs-cTnT, ng/dL & $0.010(0.009-0.012)$ & $0.012(0.010-0.015)$ & 0.15 \\
\hline Post hs-cTnT, ng/dL & $0.019(0.016-0.021)$ & $0.013(0.012-0.015)$ & $<0.01$ \\
\hline$\%$ increase of hs-cTnT, $\%$ & $67.0(46.2-90.2)$ & $8.7(0-19.3)$ & $<0.01$ \\
\hline PMI & $10(9)$ & $4(3)$ & 0.09 \\
\hline $\mathrm{TG}, \mathrm{mg} / \mathrm{dL}$ & $137.8 \pm 86.4$ & $136.0 \pm 79.4$ & 0.87 \\
\hline HDL-C, mg/dL & $56.5 \pm 16.7$ & $52.9 \pm 13.9$ & 0.08 \\
\hline LDL-C , mg/dL & $91.1 \pm 31.8$ & $103.7 \pm 33.4$ & $<0.01$ \\
\hline $\mathrm{S}-\mathrm{Cr}, \mathrm{mg} / \mathrm{dL}$ & $0.94 \pm 0.26$ & $0.91 \pm 0.33$ & 0.50 \\
\hline eGFR & $60.6 \pm 18.0$ & $64.6 \pm 25.8$ & 0.18 \\
\hline HbA1c, $\%$ & $6.3 \pm 1.0$ & $6.4 \pm 1.0$ & 0.28 \\
\hline $\mathrm{CRP}, \mathrm{mg} / \mathrm{dL}$ & $0.30 \pm 1.16$ & $0.26 \pm 0.43$ & 0.75 \\
\hline \multicolumn{4}{|l|}{ Echocardiographic data } \\
\hline LVDd, mm & $45.8 \pm 5.7$ & $46.7 \pm 6.7$ & 0.25 \\
\hline LVDs, mm & $29.9 \pm 6.5$ & $31.0 \pm 7.6$ & 0.23 \\
\hline LVEF, \% & $51.0 \pm 7.4$ & $51.2 \pm 7.5$ & 0.83 \\
\hline
\end{tabular}

Values are mean $\pm \mathrm{SD}$, median (95\% confidence interval), or $n(\%)$. BMI indicates body mass index; CKD, chronic kidney disease; OMI, old myocardial infarction; cTnT, cardiac troponin T; PMI, periprocedural myocardial injury; TG, triglyceride; HDL-C, high-density lipoprotein cholesterol; LDL, low-density lipoprotein cholesterol; S-Cr, serum creatinine; eGFR, estimated glomerular filtration rate; UCG, ultrasonic echocardiography; LVDd, left-ventricular dimension diastolic; LVDs, left-ventricular dimension systolic; and LVEF, left-ventricular ejection fraction.

(95\% CI 46.2-90.2) versus 8.7 (95\% CI 0-19.3), respectively, $P<0.01]$.

Furthermore, we compared hs-cTnT changes in patients with no significant coronary artery stenosis on CAG between this study subgroup $(n=39)$ and the control group $(n=27)$ (Table II). We assessed the level of hs-
cTnT (high hs-cTnT $>0.014 \mathrm{ng} / \mathrm{mL}$ and low hs-cTnT $\leq$ $0.014 \mathrm{ng} / \mathrm{mL}$ ) and divided into four categories according to the change of hs-cTnT level from baseline to post procedure. The incidence of "Low to High" was more frequent in this subgroup than in the control group (33\% versus $11 \%, P=0.03$ ). All three PMIs occurred in "Low 
to High" of this subgroup, which showed a much higher percentage increase of hs-cTnT compared with the control group [991.7 (95\% CI 800.0-1733.3) versus 66.7 (95\% CI 27.3-600.0), $P=0.03$ ]. However, there were no significant changes in percentage increase of hs-cTnT in other groups ("Low to Low," "High to High," "High to Low").

PMI: In this study, PMI occurred in ten (8.6\%) patients after invasive intracoronary examinations (Table III). We divided the patients into two groups by the occurrence of PMI: PMI $(n=10)$ and non-PMI $(n=105)$.

Baseline characteristics: The patients' baseline characteristics are shown in Table IV. Although there were no significant differences in sex, age, and coronary risk factors,

Table II. Serial hs-cTnT Changes in Patients with No Significant Coronary Artery Stenosis on CAG Between Study Subgroup and Control Group

\begin{tabular}{lccc}
\hline & $\begin{array}{c}\text { Study subgroup } \\
(n=39)\end{array}$ & $\begin{array}{c}\text { Control group } \\
(n=27)\end{array}$ & $P$-value \\
\hline Low to Low & $12(31)$ & $14(52)$ & 0.09 \\
Low to High & $13(33)$ & $3(11)$ & 0.03 \\
High to High & $14(36)$ & $7(26)$ & 0.39 \\
High to Low & $0(0)$ & $3(11)$ & 0.02 \\
\hline
\end{tabular}

Values are $n(\%)$ or median (95\% confidence interval). CAG indicates coronary angiography; and hs-cTnT, high-sensitivity cardiac troponin $\mathrm{T}$. the baseline hs-cTnT, post hs-cTnT, and percentage increase of hs-cTnT were significantly higher in PMI than in non-PMI [0.016 (95\% CI $0.008-0.032)$ versus 0.010 (95\% CI $0.008-0.012)$; 0.134 (95\% CI 0.072-0.188) versus 0.018 (95\% CI $0.015-0.020)$; 903.5 (95\% CI 325.01447.6) versus 54.5 (95\% CI 30.8-81.8), respectively, $P<$ 0.05]. The baseline left-ventricular diastolic dimension on ultrasonic echocardiography was significantly larger in PMI than in non-PMI $(49.4 \pm 7.2$ versus $45.4 \pm 5.4, P=$ 0.04).

Lesion and procedural characteristics: The lesion and procedural characteristics are shown in Table V. There were no significant differences between groups.

MACE: As shown in Table III, no MACE occurred during the follow-up period in PMI. However, MACE occurred in two patients $(1.7 \%)$ in non-PMI. The first patient developed sudden cardiac death 13 months after examination. He was 81 years old and had a past history of coronary artery bypass surgery for left anterior descending

Table III. The Incidence of MACE

\begin{tabular}{cccc} 
& PMI $(n=10)$ & non-PMI $(n=105)$ & Total $(n=115)$ \\
\hline MACE & $0(0)$ & $2(1.9)$ & $2(1.7)$ \\
\hline
\end{tabular}

Values are mean \pm SD or $n(\%)$. PMI indicates periprocedural myocardial injury; and MACE, major adverse cardiac event.

Table IV. Baseline Characteristics

\begin{tabular}{|c|c|c|c|}
\hline & $\begin{array}{c}\text { PMI } \\
(n=10)\end{array}$ & $\begin{array}{c}\text { non-PMI } \\
(n=105)\end{array}$ & $P$-value \\
\hline Age & $73.5 \pm 7.3$ & $71.3 \pm 9.8$ & 0.48 \\
\hline Male & $6(60)$ & $71(68)$ & 0.63 \\
\hline BMI & $24.2 \pm 1.3$ & $23.3 \pm 3.8$ & 0.43 \\
\hline Diabetes mellitus & $3(30)$ & $49(47)$ & 0.31 \\
\hline Hypertension & $7(70)$ & $82(78)$ & 0.57 \\
\hline Dyslipidemia & $7(70)$ & $76(72)$ & 0.87 \\
\hline Smoke & $7(70)$ & $61(58)$ & 0.46 \\
\hline CKD & $6(60)$ & $42(40)$ & 0.22 \\
\hline OMI & $4(40)$ & $38(36)$ & 0.81 \\
\hline HF requiring treatment & $2(20)$ & $9(9)$ & 0.24 \\
\hline \multicolumn{4}{|l|}{ Laboratory Data } \\
\hline Baseline hs-cTnT, ng/dL & $0.016(0.008-0.032)$ & $0.010(0.008-0.012)$ & 0.04 \\
\hline Post hs-cTnT, ng/dL & $0.134(0.072-0.188)$ & $0.018(0.015-0.020)$ & $<0.01$ \\
\hline$\%$ increase of hs-cTnT, $\%$ & $903.5(325.0-1447.6)$ & $54.5(30.8-81.8)$ & $<0.01$ \\
\hline $\mathrm{TG}, \mathrm{mg} / \mathrm{dL}$ & $149.6 \pm 101.5$ & $136.6 \pm 85.3$ & 0.65 \\
\hline HDL-C, mg/dL & $59.6 \pm 15.1$ & $56.3 \pm 17.1$ & 0.55 \\
\hline LDL-C, mg/dL & $86.0 \pm 28.0$ & $91.5 \pm 32.2$ & 0.60 \\
\hline $\mathrm{S}-\mathrm{Cr}, \mathrm{mg} / \mathrm{dL}$ & $1.0 \pm 0.2$ & $0.9 \pm 0.3$ & 0.22 \\
\hline eGFR & $51.9 \pm 11.6$ & $61.4 \pm 18.3$ & 0.11 \\
\hline HbAlc, \% & $5.8 \pm 0.4$ & $6.3 \pm 1.0$ & 0.10 \\
\hline $\mathrm{CRP}, \mathrm{mg} / \mathrm{dL}$ & $0.11 \pm 0.10$ & $0.32 \pm 1.22$ & 0.52 \\
\hline \multicolumn{4}{|l|}{ Echocardiographic data } \\
\hline LVDd, mm & $49.4 \pm 7.2$ & $45.4 \pm 5.4$ & 0.04 \\
\hline LVDs, mm & $32.6 \pm 9.5$ & $29.6 \pm 6.1$ & 0.18 \\
\hline LVEF, \% & $49.5 \pm 9.6$ & $51.1 \pm 7.2$ & 0.50 \\
\hline
\end{tabular}

Values are mean $\pm \mathrm{SD}$, median (95\% confidence interval), or $n(\%)$. BMI indicates body mass index; CKD, chronic kidney disease; OMI, old myocardial infarction; cTnT, cardiac troponin T; TG, triglyceride; HDL-C, high-density lipoprotein cholesterol; LDL, low-density lipoprotein cholesterol; $\mathrm{S}-\mathrm{Cr}$, serum creatinine; eGFR, estimated glomerular filtration rate; UCG, ultrasonic echocardiography; LVDd, left-ventricular dimension diastolic; LVDs, left-ventricular dimension systolic; and LVEF, left-ventricular ejection fraction. 
Table V. Lesion and Procedural Characteristics

\begin{tabular}{lccc}
\hline & PMI $(n=10)$ & non-PMI $(n=105)$ & $P$-value \\
\hline Target vessel & & & 0.29 \\
LMT & $0(0)$ & $3(3)$ & \\
LAD & $5(50)$ & $78(74)$ & \\
LCX & $3(30)$ & $27(26)$ & \\
RCA & $6(60)$ & $35(33)$ & 0.38 \\
Number of target vessel & $1.2 \pm 0.4$ & $1.3 \pm 0.5$ & 0.22 \\
Number of pull back & $2.4 \pm 1.3$ & $2.0 \pm 0.9$ & 0.59 \\
Volume of contrast media, mL & $106.5 \pm 41.1$ & $99.5 \pm 34.6$ & 0.85 \\
Stent implanted previously & & & \\
Bare-metal stent & $1(10)$ & $15(14)$ & \\
Sirolimus-eluting stent & $0(0)$ & $2(2)$ & \\
Paclitaxel-eluting stent & $0(0)$ & $3(3)$ & \\
Everolimus-eluting stent & $3(30)$ & $52(50)$ & \\
Zotarolimus-eluting stent & $2(20)$ & $21(20)$ & \\
Biolimus-eluting stent & $2(20)$ & $13(12)$ & \\
Existence of tortuosity & $1(10)$ & $30(29)$ & \\
Calcification & & & \\
None & $7(70)$ & $59(56)$ & \\
Mild & $2(20)$ & $27(26)$ & \\
Moderate & $1(10)$ & $15(14)$ & \\
Severe & $2(20)$ & $27(26)$ & \\
\hline
\end{tabular}

Values are mean \pm SD or $n(\%)$. LMT indicates left main trunk; LAD, left anterior descending artery; LCX, left circumflex artery; and RCA, right coronary artery.

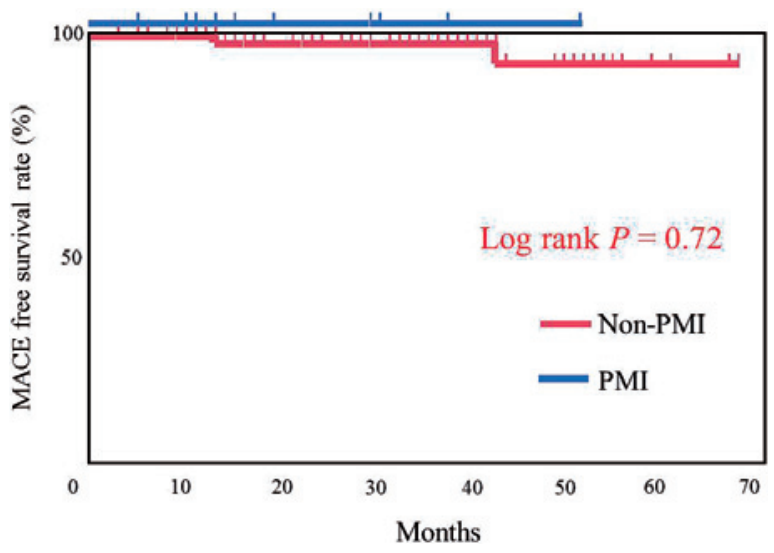

Figure 2. MACE-free survival rate. On the Kaplan-Meier analysis, MACE-free survival rate was similar between PMI and non-PMI (logrank $P=0.72$ ).

artery and PCI for right coronary artery and high-lateral branch. He was hospitalized for the treatment of pneumonia 13 months after examination. At this time, his condition changed suddenly, and he suffered cardiac arrest. Despite cardiopulmonary resuscitation, he died. We suspected that his death was due to an unexpected recurrent cardiovascular event. The second patient was hospitalized after being diagnosed with unstable angina and underwent target lesion revascularization 42 months after examination. On Kaplan-Meier analysis, there were no significant differences in the MACE-free survival rate between PMI and non-PMI (log-rank $P=0.72$, Figure 2 ).

IVUS and OCT: In this study, 75 patients underwent IVUS assessment and 40 patients underwent OCT assessment. There were no significant differences in baseline characteristics between both patients. The incidence of PMI was similar after IVUS and OCT assessments $(9.3 \%$ versus $7.5 \%, P=0.74)$. All MACEs occurred in patients who underwent IVUS assessment with non-PMI.

\section{Discussion}

The major findings of this study are as follows. First, post hs-cTnT was significantly higher in patients who underwent invasive intracoronary assessment during CAG than in those who did not. Second, PMI derived by hscTnT assay occurred in ten $(8.6 \%)$ of 115 patients after diagnostic invasive intracoronary examination without coronary revascularization. Finally, PMI after invasive intracoronary examination was not associated with MACE, and there were no significant differences in the MACEfree survival rate between PMI and non-PMI. To our knowledge, there was no study focusing on the assessment of the incidence of myocardial injury manifested by the hs-cTnT level solely after invasive intracoronary examination without PCI, making this the first study to assess the hs-cTnT level in patients having stable coronary artery disease who underwent IVUS or OCT assessment for the purposes of in-stent lesions and/or significant de novo coronary lesions during diagnostic CAG.

IVUS and OCT have recently been used more often to obtain accurate information about lesion severity and plaque morphology in the coronary arteries. We previously assessed the serum levels of hs-cTnT before and after diagnostic CAG with additional coronary assessment using IVUS in 20 patients with neither baseline hs-cTnT elevation nor renal insufficiency. ${ }^{7)}$ Age- and sex-matched patients who underwent only CAG were selected for comparison. In that study, there were no apparent complications during the procedure; however, the hs-cTnT levels in 
patients who underwent diagnostic CAG with IVUS were significantly higher. This result suggests that IVUS might cause small amounts of myocardial injury. In this study, post hs-cTnT level and percentage increase of hs-cTnT were higher in patients who underwent IVUS or OCT assessments during CAG than in those who did not. Although the absence of significant changes in the percentage increase of hs-cTnT could be due to error in serial hscTNT measurement, a very high percentage increase of hs-cTnT, particularly in patients with PMI, may indicate that IVUS or OCT impacts post procedure hs-cTnT elevation. And an imperceptible PMI derived by the hs-cTnT assay (8.6\% of all patients) occurred after diagnostic invasive intracoronary assessment.

Numerous studies to date have assessed the relationship between the baseline or post-PCI cTnT level and adverse cardiac events., ${ }^{40-16)}$ Although elevation of cTnT above the upper reference limit has been found in about $50 \%$ of patients who underwent PCI, the frequency of PMI has been more highly variable because of the various definitions of PMI. ${ }^{5}$ Some studies have shown that PMI is an independent predictor of adverse cardiac events, ${ }^{4,11-13)}$ whereas others have not. ${ }^{14-16)}$ The clinical significance of myocardial injury as manifested by elevation of the cTnT level remains controversial. In addition, there was no study to assess the relationship between cTnT level and future clinical outcome in patients who underwent invasive intracoronary assessment using IVUS or OCT during diagnostic CAG. In the present study using the hs-cTnT assay, an imperceptible PMI occurred; however, PMI was not associated with MACE. Previous studies have shown the short- and long-term safety of IVUS and OCT., ${ }^{1,2,17-22)}$ In this study, hs-cTnT elevated after invasive intracoronary assessment with IVUS or OCT usage in $8.6 \%$ of the study population. However, PMI was not associated with subsequent poor cardiac outcome. Although the study population was small, as evident in the results of this study, intracoronary examinations using IVUS and OCT were found to be safe, consistent with the results in the previous studies.

The mechanisms of hs-cTnT elevation after IVUS or OCT have not been well established. PMI during PCI is thought to be induced by distal embolization, side-branch occlusion, coronary dissection, disrupted collateral flow, and microvascular injury due to the release of vasoactive peptides. ${ }^{5,23}$ One possible mechanism of hs-cTnT elevation after invasive intracoronary examination is thrombus microembolism. Another study using computed tomography angiography reported that hs-cTnT was significantly associated with the total noncalcified plaque burden. ${ }^{24)}$ The authors concluded that silent rupture of noncalcified plaques with subsequent microembolization might be a potential source of troponin elevation. A recent study showed that the segmental plaque volume and the presence of virtual histology-derived thin-cap fibroatheroma detected by IVUS were associated with higher circulating hs-cTnT concentrations in patients with stable coronary artery disease, which might have caused subclinical plaque rupture or erosion and distal embolization. ${ }^{25)}$ Distal embolization of a microthrombus induced by IVUS or OCT might be a mechanism of hs-cTnT elevation. In this study, PMI following IVUS or OCT examination did not relate to positive remodeling or plaque burden in the observed segments. Other possible mechanisms of hs-cTnT elevation after IVUS or OCT are cardiac overload and renal insufficiency. ${ }^{26-29)}$ An increase in cardiac afterload caused by contrast media and hydration or a decrease in myocardial contractility induced by contrast media might affect myocardial injury. ${ }^{30)}$ Although the mechanisms of hs-cTnT elevation remain controversial, hs-cTnT is significantly higher in patients with renal dysfunction than those without. The present study showed no significant differences in the proportion of patients with heart failure requiring treatment or the left-ventricular ejection fraction between patients with and without PMI. However, the leftventricular diastolic dimension was significantly larger in patients with PMI than those without $(49.4 \pm 7.2$ versus $45.4 \pm 5.4, P=0.04)$. The estimated glomerular filtration rate also tended to be lower in patients with PMI than those without $(51.9 \pm 11.6$ versus $61.4 \pm 18.3, P=0.11)$. Thus, poor cardiac and renal function might be associated with hs-cTnT elevation after invasive intracoronary assessment during CAG. Although several mechanisms might be associated with hs-cTnT elevation after invasive intracoronary examination as described above, patients with PMI were not associated with subsequent poor cardiac outcomes in this study. However, this study population was small. A study with a large population is necessary to appropriately address the clinical impact of hs-cTnT assessment after IVUS or OCT.

Study limitations: This study has several limitations. First, this was a small, retrospective, and nonconsecutive study at a single center, raising the possibility of selection bias. IVUS or OCT assessment was performed at the discretion of the physician, which is another bias. Highly complex lesions might be excluded in this study population. In addition, many patients without moderate to severe renal dysfunction were excluded from this study because of lacking serial hs-cTnT data. Second, we used several types of IVUS and OCT. Myocardial injury might be affected by the catheter profile and pull-back speed. We could not evaluate the extent of myocardial injury in each imaging modality because of the small study population. Third, we defined PMI as a post-procedural hs-cTnT concentration with upper reference limit greater than fivefold, according to the "universal definition." tions of cardiac troponin elevation and PMI reported in previous studies are highly variable. In addition, the incidence of PMI using the universal definition and its influence on patient outcomes has not been established. A study with a larger population is necessary to assess the relationship between IVUS- or OCT-related myocardial injury manifested by elevations of hs-cTnT and patient outcomes.

\section{Conclusion}

An imperceptible PMI derived by hs-cTnT assay occurred after diagnostic invasive intracoronary examination. However, PMI was not associated with subsequent poor cardiac outcome. 


\section{Disclosure}

Conflicts of interest: The authors declare that they have no conflict of interest.

\section{References}

1. Guédès A, Keller PF, L'Allier PL, Lespérance J, Grégoire J, Tardif JC. Long-term safety of intravascular ultrasound in nontransplant, nonintervened, atherosclerotic coronary arteries. J Am Coll Cardiol 2005; 45: 559-64.

2. Imola F, Mallus MT, Ramazzotti V, et al. Safety and feasibility of frequency domain optical coherence tomography to guide decision making in percutaneous coronary intervention. EuroIntervention 2010; 6: 575-81.

3. Omland T, de Lemos JA, Sabatine MS, et al. A sensitive cardiac troponin $\mathrm{T}$ assay in stable coronary artery disease. N Engl J Med 2009; 361: 2538-47.

4. Testa L, Van Gaal WJ, Biondi Zoccai GG, et al. Myocardial infarction after percutaneous coronary intervention: a metaanalysis of troponin elevation applying the new universal definition. QJM 2009; 102: 369-78.

5. Prasad A, Herrmann J. Myocardial infarction due to percutaneous coronary intervention. N Engl J Med 2011; 364: 453-64.

6. Usui E, Lee T, Murai T, et al. Efficacy of multidetector computed tomography to predict periprocedural myocardial injury after percutaneous coronary intervention for chronic total occlusion. Int Heart J 2017; 58: 16-23.

7. Otsuji K, Kamezaki F, Sonoda S, et al. A rare case of myocardial infarction related to diagnostic intravascular ultrasound Heart Vessels 2013; 28: 808-13.

8. Madhavan MV, Tarigopula M, Mintz GS, Maehara A, Stone GW, Généreux P. Coronary artery calcification: pathogenesis and prognostic implications. J Am Coll Cardiol 2014; 63: 1703 14.

9. Thygesen K, Alpert JS, Jaffe AS, et al. Third universal definition of myocardial infarction. Circulation 2012; 126: 2020-35.

10. Sherwood MW, Kristin Newby L. High-sensitivity troponin assays: evidence, indications, and reasonable use. J Am Heart Assoc 2014; 3: e000403.

11. Prasad A, Singh M, Lerman A, Lennon RJ, Holmes DR Jr, Rihal CS. Isolated elevation in troponin $\mathrm{T}$ after percutaneous coronary intervention is associated with higher long-term mortality. J Am Coll Cardiol 2006; 48: 1765-70

12. Nienhuis MB, Ottervanger JP, Bilo HJ, Dikkeschei BD, Zijlstra F. Prognostic value of troponin after elective percutaneous coronary intervention: a meta-analysis. Catheter Cardiovasc Interv 2008; 71: 318-24.

13. Milani RV, Fitzgerald R, Milani JN, Lavie CJ. The impact of micro troponin leak on long-term outcomes following elective percutaneous coronary intervention. Catheter Cardiovasc Interv 2009; 74: 819-22.

14. Prasad A, Rihal CS, Lennon RJ, Singh M, Jaffe AS, Holmes DR Jr. Significance of periprocedural myonecrosis on outcomes after percutaneous coronary intervention: an analysis of preintervention and postintervention troponin $\mathrm{T}$ levels in 5487 patients. Circ Cardiovasc Interv 2008; 1: 10-9.

15. Miller WL, Garratt KN, Burritt MF, Lennon RJ, Reeder GS, Jaffe AS. Baseline troponin level: key to understanding the im- portance of post-PCI troponin elevations. Eur Heart J 2006; 27 : 1061-9.

16. Cavallini C, Verdecchia P, Savonitto S, et al. Prognostic value of isolated troponin I elevation after percutaneous coronary intervention. Circ Cardiovasc Interv 2010; 3: 431-5.

17. Hausmann D, Erbel R, Alibelli-Chemarin MJ, et al. The safety of intracoronary ultrasound. A multicenter survey of 2207 examinations. Circulation 1995; 91: 623-30.

18. Batkoff BW, Linker DT. Safety of intracoronary ultrasound: data from a multicenter European registry. Cathet Cardiovasc Diagn 1996; 38: 238-41

19. Prati F, Cera M, Ramazzotti V, Imola F, Giudice R, Albertucci M. Safety and feasibility of a new non-occlusive technique for facilitated intracoronary optical coherence tomography (OCT) acquisition in various clinical and anatomical scenarios. EuroIntervention 2007; 3: 365-70.

20. Barlis P, Gonzalo N, Di Mario C, et al. A multicentre evaluation of the safety of intracoronary optical coherence tomography. EuroIntervention 2009; 5: 90-5.

21. Taniwaki M, Radu MD, Garcia-Garcia HM, et al. Long-term safety and feasibility of three-vessel multimodality intravascular imaging in patients with ST-elevation myocardial infarction: the IBIS-4 (integrated biomarker and imaging study) substudy. Int J Cardiovasc Imaging 2015; 31: 915-26.

22. Sanuki Y, Sonoda S, Muraoka Y, et al. Contribution of poststent irregular protrusion to subsequent in-stent neoatherosclerosis after the second-generation drug-eluting stent implantation. Int Heart J 2018; 59: 307-14.

23. Cuculi F, Lim CC, Banning AP. Periprocedural myocardial injury during elective percutaneous coronary intervention: is it important and how can it be prevented? Heart 2010; 96: 736-40.

24. Korosoglou G, Lehrke S, Mueller D, et al. Determinants of troponin release in patients with stable coronary artery disease: insights from CT angiography characteristics of atherosclerotic plaque. Heart 2011; 97: 823-31.

25. Oemrawsingh RM, Cheng JM, García-García HM, et al. Highsensitivity troponin $\mathrm{T}$ in relation to coronary plaque characteristics in patients with stable coronary artery disease; results of the ATHEROREMO-IVUS study. Atherosclerosis 2016; 247: 13541.

26. Khan NA, Hemmelgarn BR, Tonelli M, Thompson CR, Levin A. Prognostic value of troponin $\mathrm{T}$ and $\mathrm{I}$ among asymptomatic patients with end-stage renal disease: a meta-analysis. Circulation 2005; 112: 3088-96.

27. Kusumoto A, Miyata M, Kubozono T, et al. Highly sensitive cardiac troponin $\mathrm{T}$ in heart failure: comparison with echocardiographic parameters and natriuretic peptides. J Cardiol 2012; 59: 202-8.

28. Tsutamoto T, Kawahara C, Yamaji M, et al. Relationship between renal function and serum cardiac troponin $\mathrm{T}$ in patients with chronic heart failure. Eur J Heart Fail 2009; 11: 653-8.

29. Freda BJ, Tang WH, Van Lente F, Peacock WF, Francis GS. Cardiac troponins in renal insufficiency: review and clinical implications. J Am Coll Cardiol 2002; 40: 2065-71.

30. Matthai WH Jr, Groh WC, Waxman HL, Kurnik PB. Adverse effects of calcium binding contrast agents in diagnostic cardiac angiography. A comparison between formulations with and without calcium binding additives. Invest Radiol 1995; 30: 6638 . 